\title{
Direct digital sensing of proteins in solution through single-molecule optofluidics
}

\author{
Georg Krainer, ${ }^{\dagger,},{ }_{l}$ Kadi L. Saar, ${ }^{\dagger,}, /\left[\right.$ William E. Arter, ${ }^{\dagger},{ }^{, /}$Raphaël P.B. Jacquat, ${ }^{\dagger}$ \\ Quentin Peter, ${ }^{\dagger}$ Pavankumar Challa,${ }^{\dagger}$ Christopher G. Taylor, ${ }^{\dagger}$ David Klenerman, ${ }^{\dagger}$ \\ Tuomas P.J. Knowles ${ }^{\dagger, *} *$ \\ ${ }^{\dagger}$ Department of Chemistry, University of Cambridge, \\ Lensfield Road, Cambridge CB2 1EW, UK \\ * Cavendish Laboratory, Department of Physics, University of Cambridge, \\ J J Thomson Ave, Cambridge CB3 OHE, UK \\ ${ }^{\text {II }}$ These authors contributed equally \\ * To whom correspondence should be addressed: \\ E-mail: tpjk2@cam.ac.uk
}




\begin{abstract}
Highly sensitive detection of proteins is of central importance to biomolecular analysis and diagnostics. Conventional protein sensing assays, such as ELISAs, remain reliant on surfaceimmobilization of target molecules and multi-step washing protocols for the removal of unbound affinity reagents. These features constrain parameter space in assay design, resulting in fundamental limitations due to the underlying thermodynamics and kinetics of the immunoprobe-analyte interaction. Here, we present a new experimental paradigm for the quantitation of protein analytes through the implementation of an immunosensor assay that operates fully in solution and realizes rapid removal of excess probe prior to detection without the need of washing steps. Our single-step optofluidic approach, termed digital immunosensor assay (DigitISA), is based on microfluidic electrophoretic separation combined with singlemolecule laser-induced fluorescence microscopy and enables calibration-free in-solution protein detection and quantification within seconds. Crucially, the solution-based nature of our assay and the resultant possibility to use arbitrarily high probe concentrations combined with its fast operation timescale enables quantitative binding of analyte molecules regardless of the capture probe affinity, opening up the possibility to use relatively weak-binding affinity reagents such as aptamers. We establish and validate the DigitISA platform by probing a biomolecular biotin-streptavidin binding complex and demonstrate its applicability to biomedical analysis by quantifying IgE-aptamer binding. We further use DigitISA to detect the presence of $\alpha$-synuclein fibrils, a biomarker for Parkinson's disease, using a low-affinity aptamer at high probe concentration. Taken together, DigitISA presents a fundamentally new route to surface-free specificity, increased sensitivity, and reduced complexity in state-of-theart protein detection and biomedical analysis.
\end{abstract}




\section{Introduction}

Sensing proteins with high specificity and sensitivity is of vital importance and remains a coveted goal in the context of biomolecular analysis and diagnostics. ${ }^{1-4}$ In contrast to nucleic acid analytes, proteins cannot be amplified directly or targeted according to base-pair complementarity, and are therefore significantly more challenging to detect effectively. ${ }^{5,6}$ Instead, to achieve high specificity and sensitivity, protein detection assays typically operate via surface-capture of target molecules by affinity reagents such as antibodies or aptamers, which isolate the target protein prior to detection through signal amplification. ${ }^{7,8}$ Foremost amongst the techniques that employ this principle are enzyme-linked immunosorbent assays (ELISAs), ${ }^{9,10}$ which rely, in their most common implementation, on the surface-capture of target molecules by a dual antibody pair in a "sandwich" complex format, followed by an enzymedriven signal amplification step (Figure 1a). A number of recent approaches have advanced the classical ELISA technique, enabling remarkable improvements in its sensitivity and throughput. ${ }^{11-14}$ This has culminated in the development of digital sensing formats such as beadbased digital ELISAs developed by Quanterix ${ }^{15}$ and methods such as those pioneered by Luminex (https://www.luminexcorp.com/), NanoString, ${ }^{16}$ and SomaSCAN, ${ }^{17}$ which display remarkable parallelization capabilities by simultaneously sensing a wide variety of protein targets.

Crucially, however, these protein sensing approaches remain reliant on surfaceimmobilization of target analyte molecules via capture probes and multi-step washing protocols for the removal of detection antibodies and reagents. These features constrain parameter space in assay design, resulting in fundamental limits in assay sensitivity due to the underlying thermodynamics and kinetics of the immunoprobe-analyte interaction (Figure 1a). Principally, the concentration of affinity capture probes that can be utilized in such approaches is limited by the finite area of the surface. The maximally achievable surface-capture probe concentrations 
in surface-based methods is $\sim 1-2 \mathrm{nM} ;{ }^{6,18}$ thus, for a capture probe with a dissociation constant $\left(K_{\mathrm{d}}\right)$ of $1 \mathrm{nM}$, as is typical of many affinity reagents, only $50 \%$ of an analyte will be bound to the probe, which falls to $<1 \%$ for capture probes with $K_{\mathrm{d}}>100 \mathrm{nM}$ (Figure 1a,b(i)). This low capture efficiency is exacerbated by dissociation of the probe-analyte complex (particularly for $\left.K_{\mathrm{d}}>1 \mathrm{nM}\right)$ as the binding equilibrium is disturbed over tens of minutes to hours by the washing steps required by the ELISA or bead-based protein detection assays; hence, off-rates become significant (Figure 1a,b(ii)). These lengthy assay workflows thus present challenges in maintaining probe-analyte interactions under non-equilibrium conditions. Together, these effects constitute intrinsic drawbacks to surface-based methods, resulting in the limitation that highly optimized capture probes with sub-nanomolar dissociation constants are required for effective sensing, and that a calibration step is needed that converts the recorded signal to the actual target concentration.

Overcoming these limitations requires a fundamentally different assay design and, conceptually, involves the implementation and combination of two essential, yet so far unexploited elements. Firstly, analyte capture needs to be conducted in the presence of arbitrarily high concentrations of the affinity capture probe. This feature ensures nearquantitative binding of the target molecule (Figure 1b,c), and can be achieved, for instance, by performing the assay in solution. Secondly, excess probe removal needs to be suitably fast (i.e., on timescales much faster than the half-time of the probe-analyte dissociation). In this way, sensing can take place before the system re-equilibrates and the probe-analyte complex dissociates (Figure 1b,c). Together, these two features - the possibility to use arbitrarily high probe concentrations in solution and to remove unbound probe in a rapid manner-would allow the fundamental drawbacks of incumbent surface-based sensing approaches to be overcome. 


\section{a}

$C_{\text {probe }} \lessgtr 1-2 \mathrm{nM}$
very low
capture
efficiency
for $K_{\mathrm{d}}>C_{\text {probe }}$
panel b(i)

panel $b(i)$

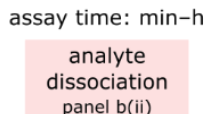

panel b(ii)

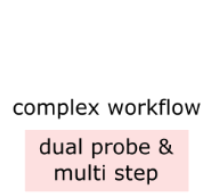
multi step surface-based

immunoassay

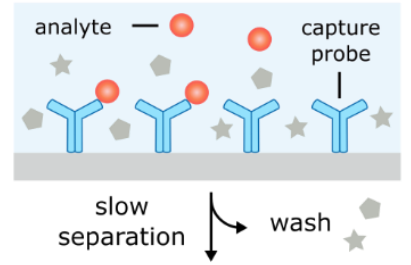

O

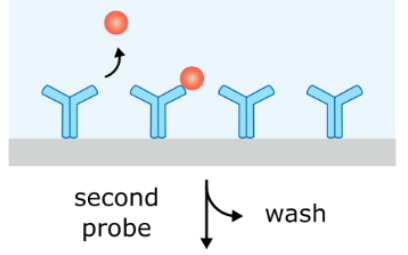

detection

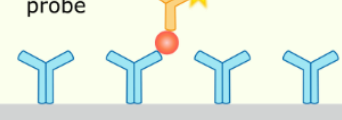

C

\section{free-solution}

immunoassay
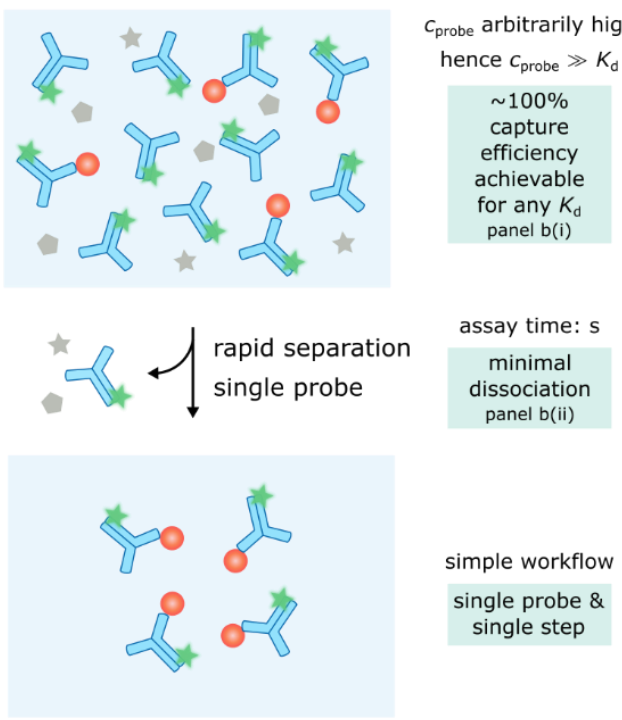

simple workflow

single probe \&

single step

b

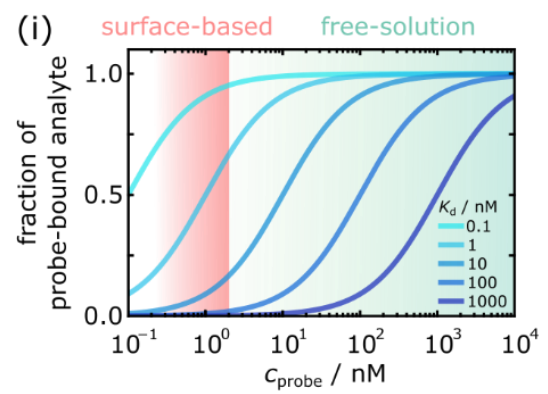

(ii)

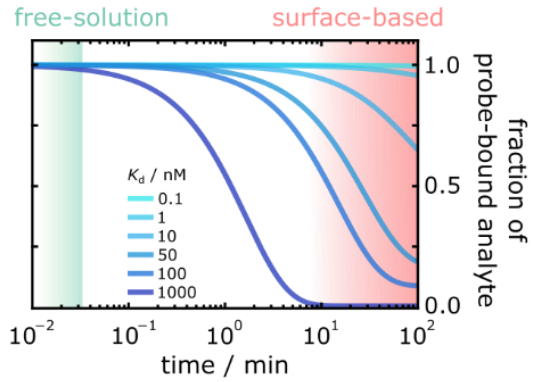

Figure 1: Immunosensing in free solution overcomes fundamental limitations of surface-based sensing methods. (a) Schematic of surface-based immunosensor assays (e.g., ELISAs or bead-based assays) and their inherent limitations in terms of capture efficiency, analyte dissociation, and workflow complexity. Conventional methods are limited to surface-capture probe concentrations $\left(c_{\text {probe }}\right)$ in the low nanomolar regime (i.e., 1-2 nM). Under these conditions, a significant amount of the analyte is not bound and thus remains undetected, especially when using affinity probes with $K_{\mathrm{d}}>1 \mathrm{nM}$ (see panel $b(i)$ ). Additionally, the binding equilibrium is disturbed during washing steps that take tens of minutes to several hours. Hence, dissociation of the immunoprobe-analyte complex becomes significant, particularly for weak-binding affinity probes with $K_{\mathrm{d}}>1 \mathrm{nM}$ (see panel b(ii)). Moreover, conventional assays involve multi-step procedures and typically require additional affinity probes for detection. (b) Speciation curves depicting fraction of probe-bound analyte versus affinity probe concentration (panel b(i)) and fraction of probe-bound analyte versus time (panel b(ii)). Shown are simulations for probe-analyte affinities with $K_{\mathrm{d}}=0.1-1000 \mathrm{nM}$ (from light to dark blue). Turquoise and red shaded areas denote operation regimes. In panel $\mathrm{b}(\mathrm{ii}), t=0$ refers to the moment when the unbound probe is removed from the system. Details on the simulations are given in the Supplementary Information. (c) Schematic illustration depicting the principles of a free-solution immunosensor assay and its advantages over conventional surface-based methods. By performing the immunosensor reaction in 
solution, arbitrarily high concentrations of the affinity probe can be used, which permits quantitative antigen binding, even for affinity reagents with $K_{\mathrm{d}}>1 \mathrm{nM}$ (see panel b(i)). A rapid timescale for the removal of non-target bound probe prevents the system from re-equilibrating (see panel b(ii)) and sets the basis for quantitative analysis of the immunoprobe-analyte complex interaction. Additionally, the assay can be accomplished in a single step and requires only a single affinity reagent.

Here, we present an approach, termed digital immunosensor assay (DigitISA), that implements the principles of in-solution analyte capture and fast removal of excess probe and allows protein targets to be detected and their abundance to be quantified in solution without the need of washing steps. We achieve this objective by exploiting free-flow electrophoretic separation which, crucially, allows protein-bound affinity reagents to be discriminated from non-protein bound ones based on a difference in their electrophoretic mobility (i.e., the ratio of the net electrical charge of a molecule to its size) on a rapid, second timescale (Figure 2a). To achieve a high detection sensitivity, we combine the electrophoretic separation step with singlemolecule detection by laser-induced fluorescence confocal microscopy, which allows the number of protein-bound affinity reagent molecules present in the sample to be detected through single-molecule counting. In addition to the high sensitivity, the digital nature of the detection process allows for its calibration-free use.

Because DigitISA operates in free solution and allows for separation of excess probe without the need for washing steps (Table 1), arbitrarily high concentrations of binding probes can be employed, enabling the probe-target binding equilibrium to be favorably manipulated to optimize target capture efficiency (Figure 1b,c). Furthermore, the assay operates on a fast timescale $(\sim 2 s)$, meaning that the probe-analyte binding interaction is maintained during the entire sensing process (Figure 1b,c). Combined, these factors allow the use of relatively weakbinding capture reagents $\left(K_{\mathrm{d}}=10-1000 \mathrm{nM}\right)$, whereas conventional approaches require highlyoptimized probes with sub-nanomolar affinity. This finding is significant, as the development of ultra-high-affinity antibody probes is a costly and non-trivial process, and may not be feasible 
for some biomarker targets, such as highly heterogenous biomolecular complexes, including protein aggregation species. ${ }^{19,20}$ Therefore, our assay opens up the possibility to use commonly overlooked affinity reagents, such as aptamers, in quantitative single-molecule sensing. Moreover, by incorporating microchip electrophoresis in addition to affinity selection, DigitISA provides an additional criterion for signal generation to afford highly specific protein sensing; this enhances the selectivity in protein sensing. ${ }^{21}$ Finally, the surface-free nature of the assay not only reduces false-positive signaling by non-specific surface adsorption, it also allows the assay to be operated in a single step. Given that DigitISA uses only a single affinity reagent per target, the complexity of assay design is reduced because validated, noncross-reactive affinity probe pairs and multi-epitopic targets are not required.

Table 1: Comparison between conventional surface-based immunosensor assays for protein detection and the digital immunosensor assay (DigitISA) developed here.

\section{ELISA and bead-based immunoassays}

Surface-based

Capture efficiency determined by $K_{\mathrm{d}}$ and surface concentration of affinity probe Multi-step assay involving washing steps for removal of excess probes and reagents

Specificity from affinity criterion only Affinity reagent pair

\section{Digital immunosensor assay (DigitISA)}

Free solution

Complete binding of target molecules achievable even with low affinity probes

Single-step assay with fast removal of excess affinity probe Specificity from combined affinity and electrophoretic criteria Single affinity reagent 

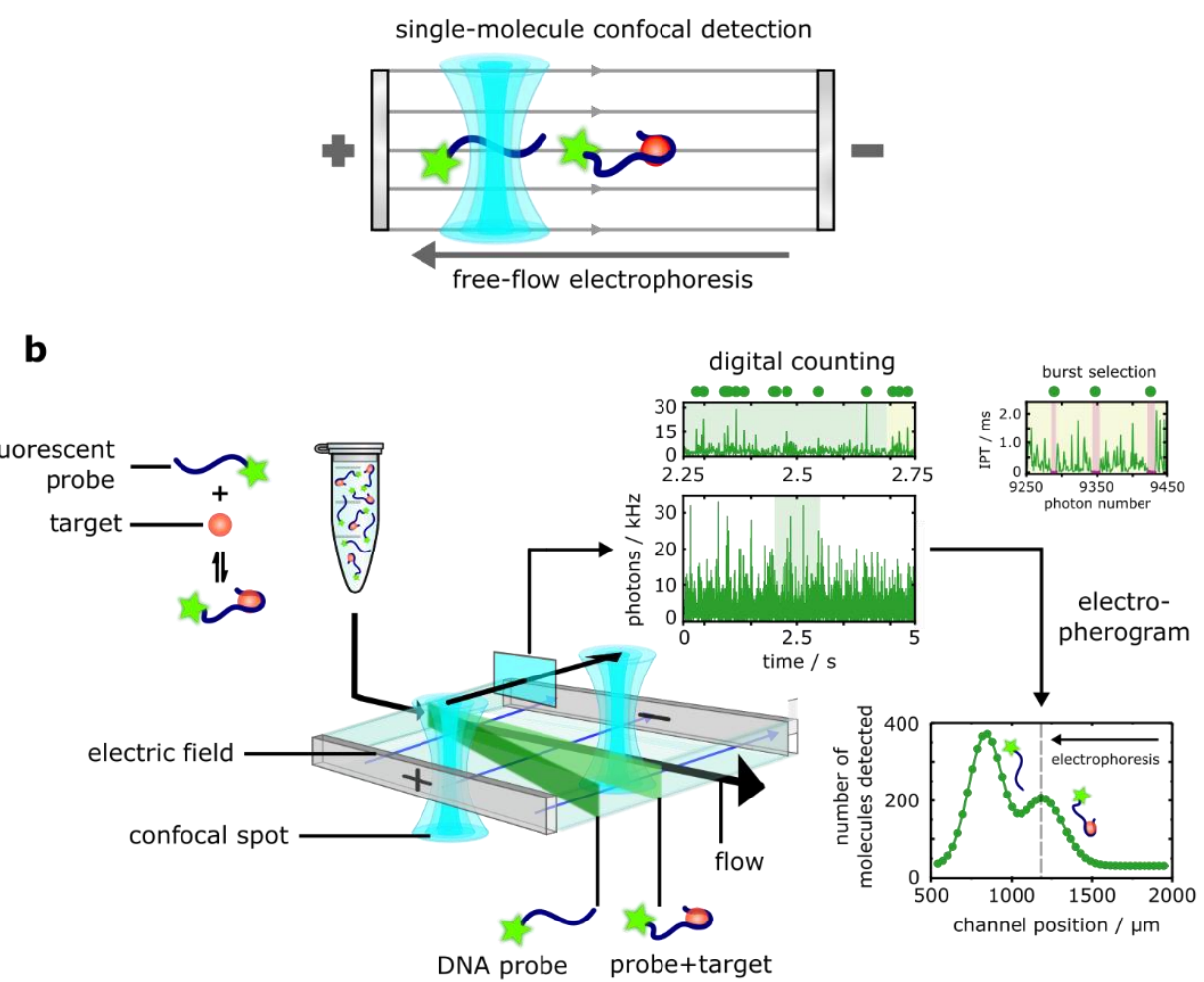

C

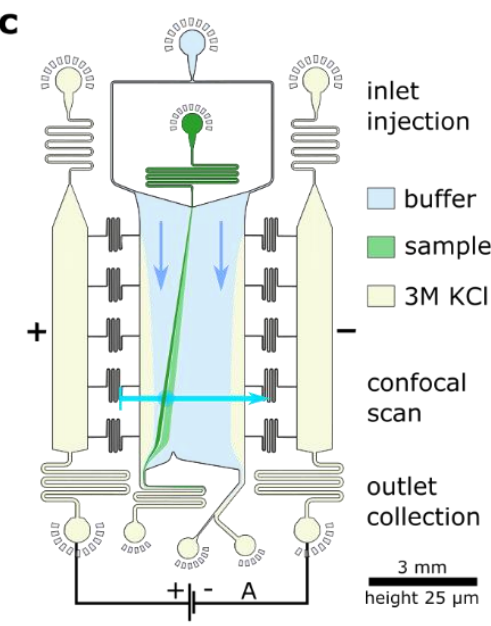

d
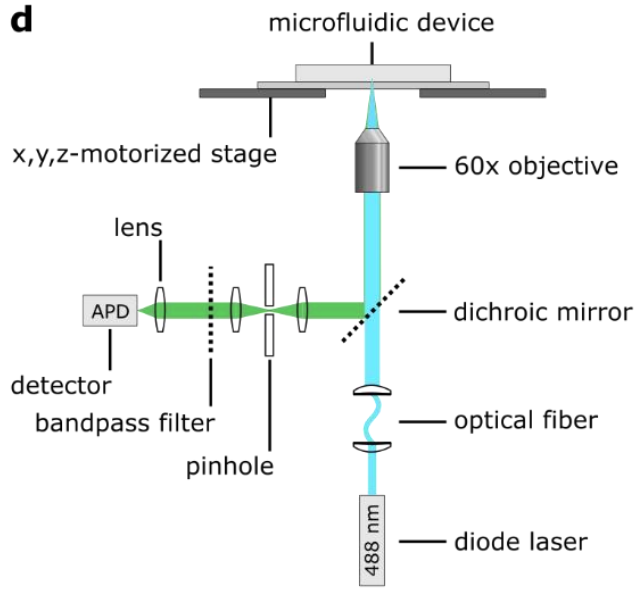

Figure 2: Working principle of DigitISA and its implementation. (a) Illustration of the DigitISA concept. DigitISA integrates electrophoretic separation and single-molecule detection in a platform for single-step sensing of target proteins in solution using only a single affinity reagent. (b) Schematic of the experimental workflow. The sample, including a mixture of the target protein and its fluorescently labeled probe, is injected into a micron-scale electrophoretic separation unit. The application of an electric field allows protein-bound probe molecules to be discriminated from those probe molecules that are not bound to the protein target, owing to a difference in their electrophoretic mobilities. Confocal scanning across the separation chamber is performed and the number of molecules traversing the confocal volume at each of the scanned positions is estimated 'digitally' from the recorded photon-count time trace using a combined inter-photon time (IPT) and photon-count threshold burst-search algorithm. 
From the obtained counts an electropherogram is created allowing for a discrimination between proteinbound affinity probe and free probe. (c) Design of the free-flow electrophoresis device. Sample is flown into the microfluidic chip by the central injection port where it is then surrounded by the carrier buffer solution. The electrophoresis chamber is connected to a co-flowing electrolyte solution $(3 \mathrm{M} \mathrm{KCl})$ via ridges, which allows for a narrow sheet of electrolyte to flow along the sides of the chamber. An electric field is applied from metal clips at the outlets of the electrolyte channels, which propagates along the electrolyte sheet and enables separation of molecules perpendicular to the flow direction. (d) Schematic of the confocal microscopy setup used for single-molecule detection. A diode laser is used to excite the sample through an objective, and a single-photon counting avalanche photodiode (APD) is used to register emitted photons from the sample. The confocal spot is moved across the cross-section of the chip in a stepwise manner with the aid of a motorized stage. This allows the flux of the protein-bound probe molecules to be estimated. Details of the setup are described in the Methods section.

\section{Results and Discussion}

\section{Working principle and assay design}

The working principle and experimental implementation of the DigitISA platform is depicted in Figure $2 b-d$. Sample containing the target protein and a fluorescently labeled capture probe is injected into the microfluidic free-flow electrophoresis separation chip fabricated in poly(dimethylsiloxane) (PDMS) (see Methods). The sample stream, upon its entry into the separation region, is surrounded by a buffered carrier medium, so that the sample forms a narrow stream at the center of the chamber. To discriminate the free probe from its analyte-bound form, an electric potential is applied perpendicular to the flow direction using co-flowing electrolyte solutions that act as liquid electrodes and ensure the application of stable electric fields, as described earlier. ${ }^{22,23}$ Simultaneously, laser-induced confocal fluorescence microscopy is used to detect individual molecules by scanning the confocal volume across the microfluidic chip. Notably, scanning is performed in a stepwise manner at the mid-height of the channel at a distance of $4 \mathrm{~mm}$ downstream from the position where the sample first entered the electric field. The number of molecules traversing the confocal volume at each of the scanned positions is estimated from the recorded photon-count time trace using a combined inter-photon time (IPT) 
and photon-count threshold burst-search algorithm (see Methods). This approach has been shown to enable effective discrimination between photons that originate from single fluorescent molecules and those that correspond to a background, ${ }^{25,26}$ thus allowing individual molecules to be counted directly, that is, in a digital manner. From the detected number of molecules at each position, an electropherogram is generated by plotting the obtained single-molecule counts as a function of chip position. Thereby, the separation between protein-bound affinity probe and free probe can be visualized, and the concentration of the target complex quantified.

\section{Sensing of a streptavidin-biotin affinity complex}

To demonstrate the possibility of using the combined free-flow electrophoresis and singlemolecule counting DigitISA platform for biomolecular detection and quantification, we set out to probe the formation of a biotin-streptavidin complex. Specifically, we investigated the binding of a biotinylated and fluorophore-conjugated DNA sequence to monovalent streptavidin (Figure 3a). This interaction mimics the binding of a protein molecule to its affinity reagent with the binding interaction being very well defined and of high affinity. ${ }^{24}$

We first examined whether an applied electric field allows for a discrimination between the streptavidin-bound and unbound DNA probe according to a difference in electrophoretic mobility. To this end, we incubated $25 \mathrm{pM}$ of the monovalent streptavidin with $50 \mathrm{pM}$ of the biotinylated probe DNA and injected the sample into the free-flow electrophoresis chip by applying an electric potential of $150 \mathrm{~V}$. Additionally, we injected a control sample including only the biotinylated DNA with no streptavidin added into a different identically fabricated chip. 5-second-long step-scans along the cross-section of the microfluidic separation chamber were performed for both samples and the number of molecules traversing the confocal volume at each of the scanned positions was estimated burst analysis of photon time traces (see Methods). Using the obtained single-molecule counts, electropherograms across the crosssection of the separation chamber were obtained for the two samples (Figure 3b). From these 
data, we observed that the binding of streptavidin decreased the electrophoretic mobility of the DNA-conjugated biotin molecules in comparison to the free DNA-conjugated biotin probe, with the free biotin eluting at a channel position of $x=500 \mu \mathrm{m}$ and the streptavidin-biotin complex at $x=750 \mu \mathrm{m}$ (red line). Indeed, at the former position, only a minimal elution of fluorescent molecules occurred for the control sample (blue line), indicating that this elution position corresponds to that of the biotin-streptavidin complex. Moreover, we also noted that the recorded fluorescence at the position where the unbound biotin-DNA molecules eluted was higher in the control sample than for the case when the streptavidin target was present. This observation further confirmed the integration of biotinylated DNA molecules into the complex.

a

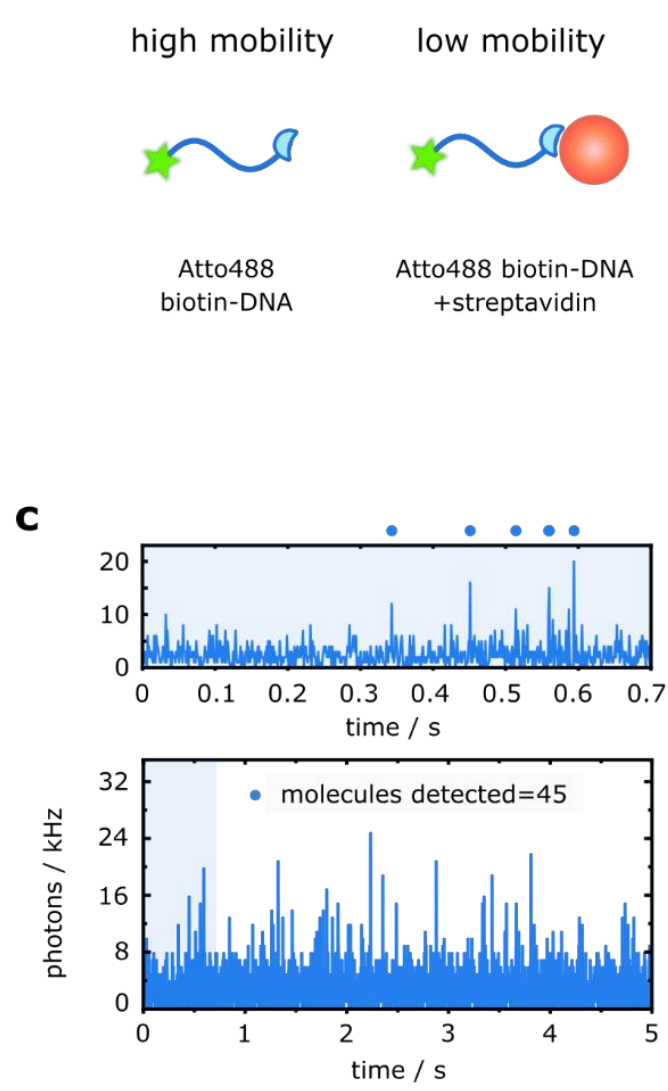

b
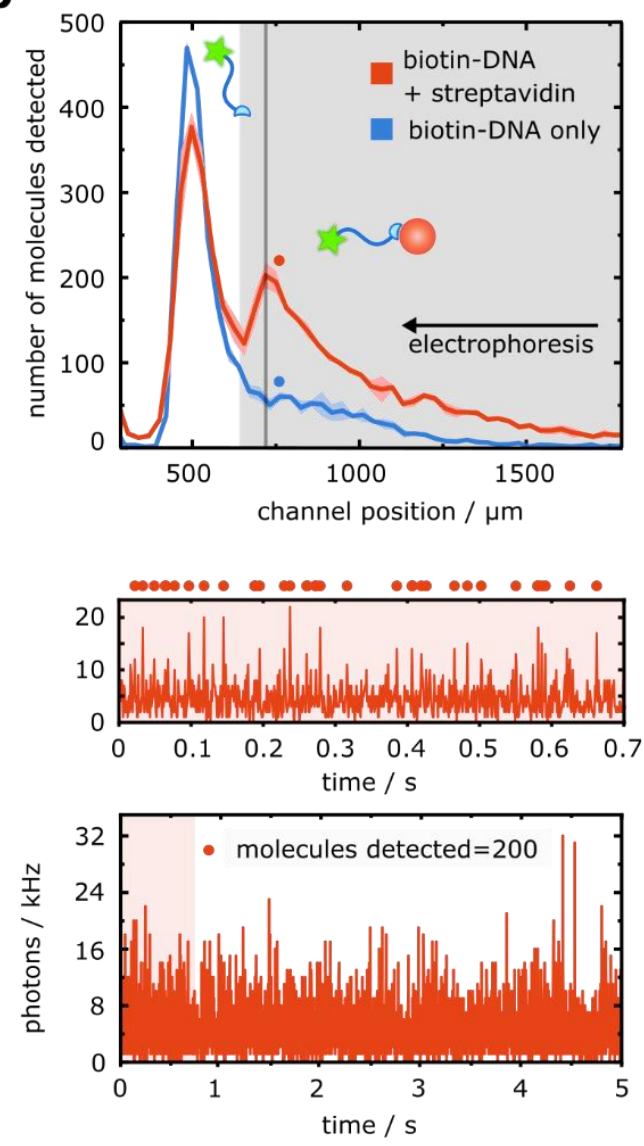

Figure 3: Sensing of a biotin-streptavidin complex using the DigitISA platform. (a) The binding of monovalent streptavidin to a biotinylated and fluorophore-conjugated DNA sequence was studied. Binding of the streptavidin species reduces the electrophoretic mobility of biotinylated DNA probe. (b) Electropherogram as obtained by stepwise scanning the confocal volume across the cross-section of 
the channel for the biotin-streptavidin mixture (red line; average of $N=3$ repeats, the shaded bands correspond to the standard deviation) and for the control sample (blue line) at the mid-height of the channel, demonstrating the presence and separation of both streptavidin-bound and non-bound biotinylated DNA molecules in the sample. The region shaded in light grey was used to extract the number of streptavidin-biotin complexes that passed the device in a given time, and ultimately, its concentration (see main text). (c) Exemplary photon-count time traces for the control sample (left panel, blue) and the mixture (right panel, red) at the position where the concentration of the complex molecules was the highest as indicated with colored dots in panel $b$. The number of molecules at each of the scanned positions was estimated using a burst-search algorithm as detailed in the Methods section. Time traces in the panels above are zoom-in views with dots indicating detected single-molecule events. The bin time was $1 \mathrm{~ms}$ in all traces.

Having confirmed the capability of the platform to detect the formation of the biotinstreptavidin complex, we next set out to explore its potential to estimate absolute concentrations. Indeed, while many protein quantification assays, including conventional ELISAs, rely on a calibration curve between the observed signal and the concentration of the analyte molecules, the platform developed here counts the number of passing molecules in a digital manner, and therefore has the potential to estimate target concentrations without the requirement for calibration.

To explore this opportunity, we evaluated the number of molecules that eluted between channel positions of $x=700 \mu \mathrm{m}$ and $x=2200 \mu \mathrm{m}$ (Figure 3b, region shaded in light grey). We recorded $\bar{n}_{\text {mixture }}=2391 \pm 37$ molecules (mean $(\bar{n}) \pm$ standard deviation $(s)$ of $N=3$ repeats) for the biotin-streptavidin mixture (red line) in contrast to only $\bar{n}_{\text {control }}=789 \pm 30$ molecules for the control sample (blue line). The non-zero count for the control sample is likely to originate from impurities in the biotin-DNA sample or from degraded forms of the DNA. The difference between the two counts, $\bar{n}_{\text {complex }}=\bar{n}_{\text {mixture }}-\bar{n}_{\text {control }}=1601 \pm 48$ molecules, can be attributed to streptavidin-biotin complexes. We note that this count corresponds to the molecular flux in the regions where the single-molecule time traces were recorded. The flux of the streptavidin-biotin 
complex molecules through the full device, $F_{\text {total }}$, can be estimated from the following relationship:

$$
\begin{aligned}
F_{\text {total }} & =\frac{\bar{n}_{\text {complex }}}{t} \cdot \frac{h \cdot d_{\text {step }}}{\frac{\pi}{4} \cdot z \cdot w}=\frac{(1601 \pm 48) \text { molecules }}{5 \mathrm{~s}} \cdot \frac{28 \mu \mathrm{m} \cdot 31.7 \mu \mathrm{m}}{\frac{\pi}{4} \cdot 3 \mu \mathrm{m} \cdot 0.4 \mu \mathrm{m}} \\
& =298000 \pm 9000 \text { molecules s }^{-1}
\end{aligned}
$$

where $t$ is the time period over which the time traces were recorded, $h$ is the height to which the separation chamber was fabricated, $d_{\text {step }}$ is the step size at which the single-molecule time traces were recorded, and $z$ and $w$ are the width and the height of the confocal detection volume, respectively, describing its cross-section. The latter two parameters were estimated from a fluorescent correlation spectroscopy (FCS) measurement (see Methods) and were determined to be $z=3 \mu \mathrm{m}$ and $w=0.4 \mu \mathrm{m}$ for our setup. As the sample was entering the device at a flow rate of $Q_{\text {sample }}=70 \mu \mathrm{L} \mathrm{h}^{-1}$, this molecular flux yielded an estimate of

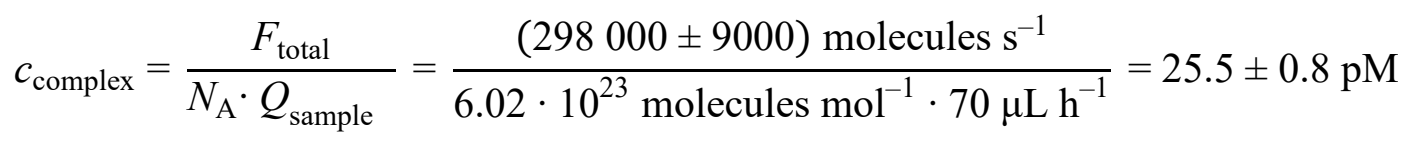

for the concentration of the streptavidin-biotin complex in the sample, with $N_{\mathrm{A}}$ being the Avogadro constant. The binding affinity between the monovalent streptavidin and biotin molecules has previously been estimated to be in the femtomolar range. ${ }^{24}$ As such, under the conditions used here (25 pM streptavidin and $50 \mathrm{pM}$ biotin), we would indeed expect all of the monovalent streptavidin in the mixture to be incorporated into the complex. This result thus elegantly exemplifies that the platform can be used to obtain direct calibration-free readouts of molecular concentrations.

With the control sample including $\bar{n}_{\text {control }}=789 \pm 30$ molecules that eluted in the detection region, we estimate the limit of detection ( $\left.\mathrm{LOD}=\bar{n}+3 \cdot s_{\text {control }}\right)$ for the assay to be 
$789+3 \cdot 30=879$ molecules, defined as three standard deviations above the background count of the control sample. Converting this flux into concentrations (Eq. 2), we conclude the LOD to be $1.5 \mathrm{pM}$. We note that this limit is determined by the non-complete baseline separation of the bound and non-probe and, hence, LOD could be enhanced by further optimizing the resolution of the separation unit.

\section{Immunosensing of the protein biomarker immunoglobulin $\mathbf{E}$}

Having established and validated the DigitISA platform for highly sensitive detection and quantification of biomolecular complexes, we set out to demonstrate the advantage of the fast (i.e., $2 \mathrm{~s}$ ) assay timescale in the context of quantitative protein biomarker sensing. We investigated the protein immunoglobulin $\mathrm{E}(\operatorname{IgE})$, which is a key component of the human immune system. IgE shows a particular relevance in allergic responses, and elevated $\operatorname{IgE}$ concentrations are a defining characteristic of hyper-IgE syndrome and IgE myeloma. ${ }^{27,28} \mathrm{We}$ used an established IgE aptamer ${ }^{29}$ labeled with Atto488 fluorophore (see Methods) to detect the presence of IgE molecules (Figure 4a). Aptamers are considered an attractive class of affinity reagents because they can be quickly and easily generated by in vitro evolution such as 'systematic evolution of ligands by exponential enrichment' (SELEX), ${ }^{30-32}$ and are readily produced by chemical synthesis while offering recognition capabilities that rival those of antibodies. Importantly, their relatively small physical size in comparison to full antibodies permits a more significant alteration in their electrophoretic mobility upon binding to a target, setting the basis for an efficient electrophoretic separation between the protein bound and nonbound forms of the molecule.

Crucially, however, most conventional aptamers comprised of natural nucleotides (as opposed to the hydrophobically-modified bases employed in SOMAmer reagents) ${ }^{17,33}$ are limited in binding strength, with typical values of $K_{\mathrm{d}}>1 \mathrm{nM}$. Thus, quantitative sensing by aptamer probes in surface-based immunoassays is hindered by the relatively fast rate of probe- 
analyte dissociation, which has limited their widespread use, despite their many advantages. For example, with the IgE aptamer employed here $\left(K_{\mathrm{d}} \approx 50 \mathrm{nM}\right),{ }^{34,35}$ during an experiment time of 45 minutes as required for a 'fast' aptamer-based ELISA assay, ${ }^{36}$ approximately $66 \%$ of aptamer-analyte complexes present in the initial aptamer-analyte binding equilibrium would dissociate and not contribute to the sensing signal (Figure 1b(ii)). Since DigitISA operates more than two orders of magnitude faster than ELISA-based approaches, negligible probe-analyte dissociation occurs on the assay timescale (Figure $1 \mathrm{~b}(\mathrm{ii})$ ). Thus, the quantity of the aptamerprobe complex, probed by DigitISA, accurately reflects the equilibrium concentration of the complex present in the original probe-analyte mixture. Therefore, for an affinity probe of known $K_{\mathrm{d}}$, our method allows quantitative protein sensing in a single, calibration-free measurement, even at concentrations far below the $K_{\mathrm{d}}$ of the interactions.

a
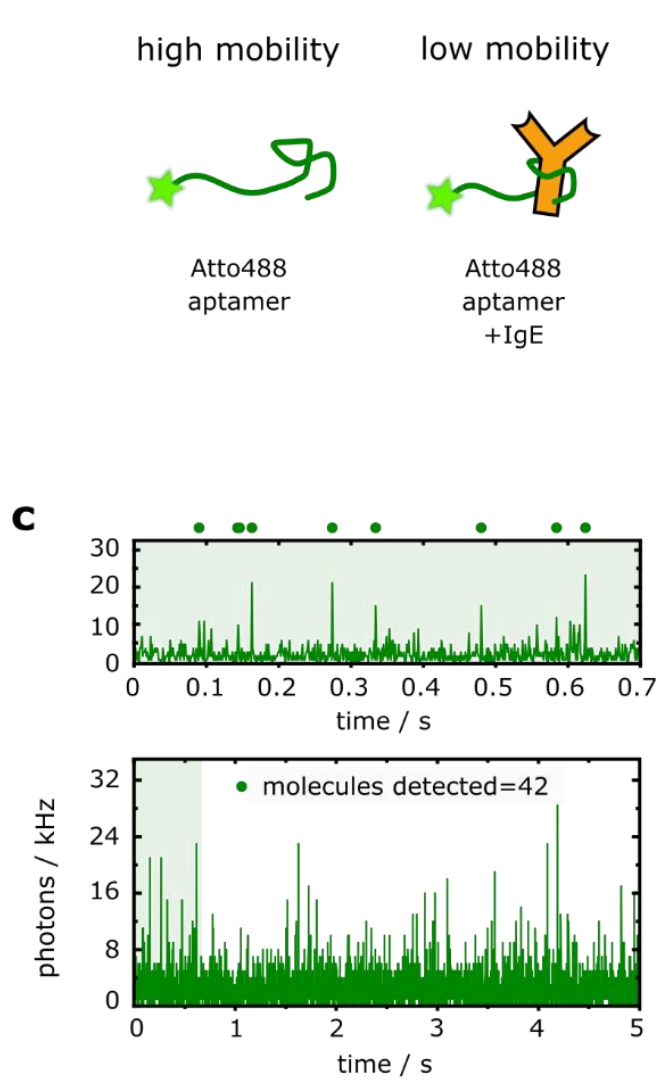

b
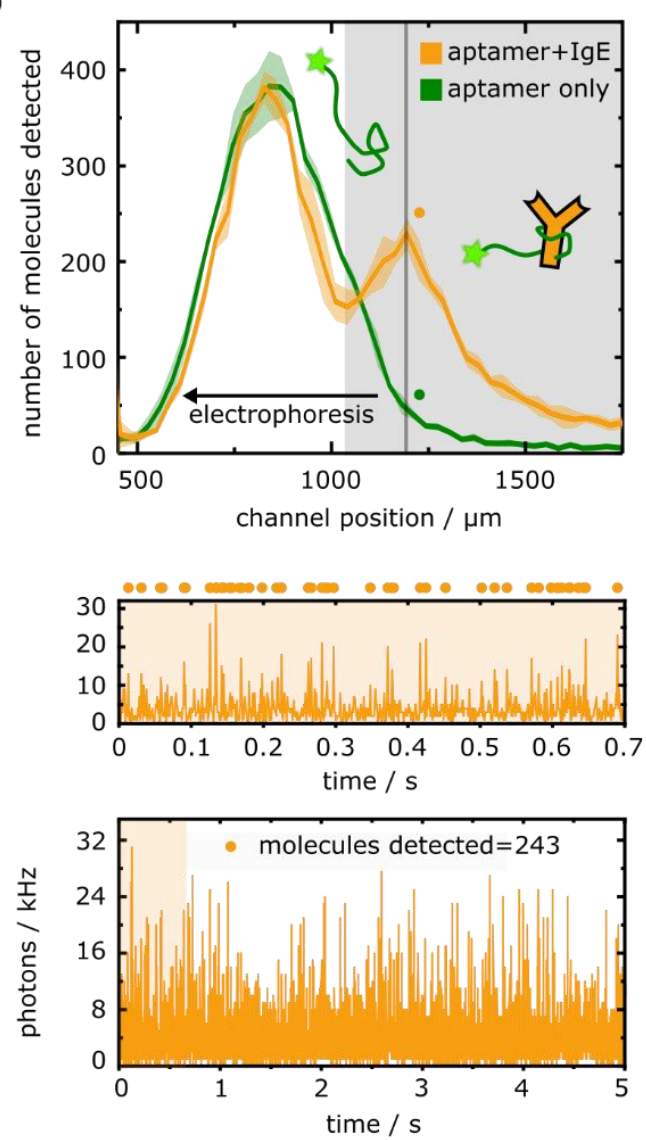

Figure 4: DigitISA immunosensing of IgE with an aptamer probe. (a) The binding of an IgE-aptamer probe to its target IgE reduces the electrophoretic mobility of the probe, allowing for fast electrophoretic 
separation of the aptamer probe from the immuno-complex for subsequent confocal detection. (b) Electropherogram as obtained by stepwise scanning of the confocal volume across the cross-section of the channel for the IgE-aptamer sample (orange line; average of $N=3$ repeats, the shaded bands correspond to the standard deviation) and for the free aptamer probe (green line). The region shaded in light grey was used to quantify the concentration of IgE by monitoring the flux of fluorescent molecules in the region shaded in light grey (see main text). (c) Exemplary photon-count time traces for the control sample (left panel, green) and the mixture (right panel, orange) at the position where the concentration of the complex molecules was the highest as indicated with colored dots in panel $b$. The number of molecules at each of the scanned positions was estimated using a burst-search algorithm as detailed in the Methods section. Time traces in the panels above are zoom-in views with dots indicating detected single-molecule events. The bin time was $1 \mathrm{~ms}$ in all traces.

To demonstrate this principle, a mixture of $\operatorname{IgE}(40 \mathrm{nM})$ and aptamer probe $(50 \mathrm{pM})$ was premixed and injected into the microfluidic separation device and an electropherogram acquired (Figure $4 b$, orange line) in a similar manner to the streptavidin-biotin system described above. These concentrations were chosen so that only a small proportion of protein would be bound by the probe, to test whether our approach would allow effective back-calculation of the true protein concentration. As expected, both the free probe and the probe-protein complex were observed in the electropherogram with the complex eluting at a higher channel coordinate (smaller deflected distance) due to its reduced mobility (Figure 4b, orange line). A control experiment with no IgE showed only the free-probe peak with a minimal amount of fluorescence detected at the elution position of the complex (Figure 4b, green line). As before, we used the photon-count time traces to estimate the flux of fluorescent molecules in the regions where the complex eluted (Figure 4b, region shaded in light grey). From these data, we concluded that $\bar{n}_{\text {mixture }}=2847 \pm 62$ molecules eluted over a time period of $5 \mathrm{~s}$ for the mixture and $\bar{n}_{\text {control }}=856 \pm 29$ molecules for the control sample that included only the probe molecules without the target. Using a conversion strategy similar to what was described above for the biotin-streptavidin system, this molecular flux value yielded an estimate of $c_{\text {complex }}=21.7 \pm 0.7 \mathrm{pM}$ for the concentration of the aptamer-IgE complex. By a similar 
argument as presented earlier for the streptavidin-biotin system, we estimated the LOD of detected probe-protein complex to be $0.9 \mathrm{pM}$.

Using a simple 1:1 binding model for the aptamer-IgE interaction and our knowledge of the concentrations of the $\operatorname{IgE}$ bound $\left(c_{\text {complex }}=21.7 \mathrm{pM}\right)$ and free $\left(c_{\text {free }}=50 \mathrm{pM}-\right.$ $21.7 \mathrm{pM}=28.3 \mathrm{pM}$ ) forms of the aptamer probe, we calculated the concentration of $\operatorname{IgE}$ present in the sample to be $c_{\mathrm{IgE}}=38.4 \pm 1.6 \mathrm{nM}$ according to $c_{\mathrm{IgE}}=c_{\text {complex }} \cdot\left(K_{\mathrm{d}}+c_{\text {free }}\right) / c_{\text {free }}$. This value showed excellent agreement with the nominal starting concentration of $\operatorname{IgE}$, with the small deviation likely originating from an uncertainty in the reported value of the binding constant $K_{\mathrm{d} .}{ }^{34,35}$ This result demonstrates the efficacy of our approach in quantitative protein sensing even for relatively weak-binding probes, as enabled by the rapid fractionation of protein bound and unbound probe by the microchip free-flow electrophoresis DigitISA platform, and provides a route to performing measurements in a regime where the availability of the affinity reagent is limited. Notably, our approach exploits the fact that detection relies only on a single, monovalent interaction between the probe and the analyte, which allows facile back-calculation of the target concentration from the underlying binary equilibrium, as demonstrated above. Conversely, even for a well-characterized ELISA experiment, such calculation is challenging to realize given the multiple analyte-antibody equilibria that are present in the sandwich-type formats employed in ELISAs.

\section{Sensing of $\alpha$-synuclein amyloid fibrils}

Having shown the advantages associated with the fast assay timescale, we next demonstrated the ability of the free-solution DigitISA assay to directly sense the probe-analyte binding equilibrium by the use of high probe concentration. To do so, we investigated the binding between $\alpha$-synuclein fibrils and an aptamer which has been shown previously to weakly bind fibrillar forms of the $\alpha$-synuclein protein with an approximate $K_{\mathrm{d}}$ of $500-1000 \mathrm{nM} .{ }^{37}$ Fibrillar $\alpha$-synuclein is a molecular hallmark of Parkinson's disease and other synucleinopathies; sensing 
of $\alpha$-synuclein aggregates is thus proposed as a means for the early detection for these conditions. ${ }^{38}$ As illustrated in Figure 5a, binding of the aptamer to the $\alpha$-synuclein fibrils is expected to suppress the electrophoretic mobility of the fibrils, thus enabling efficient separation of aptamer-bound fibrils from the unbound probe that is provided in excess.

a

high mobility low mobility
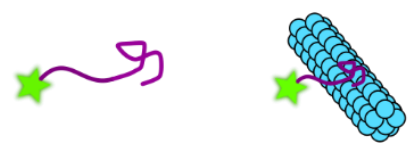

Alexa488

b
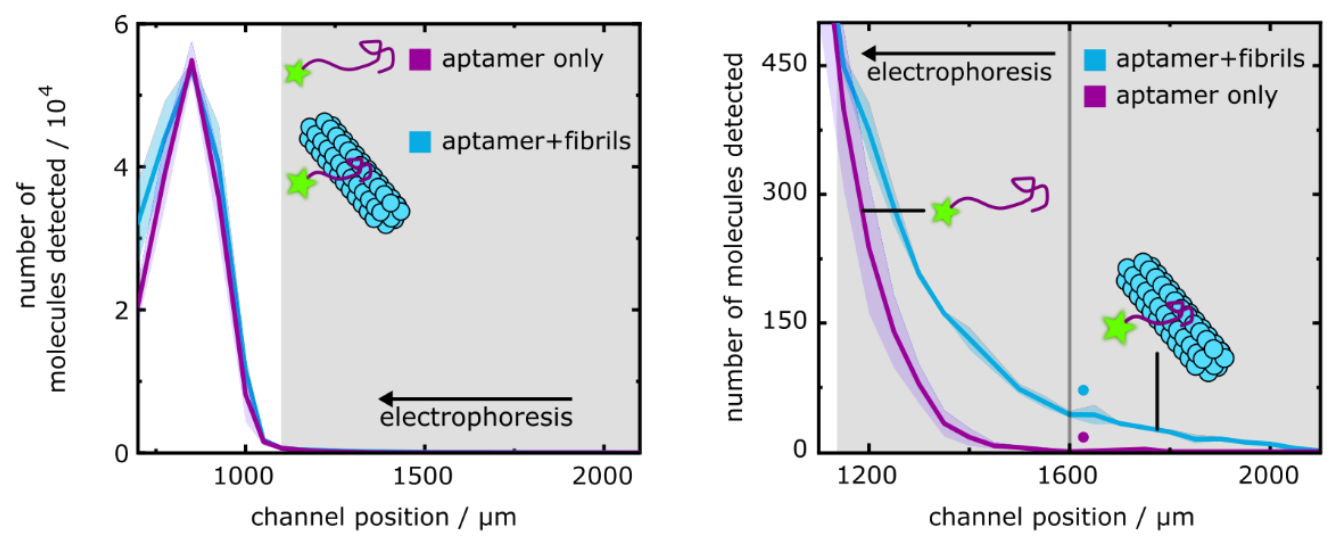

C
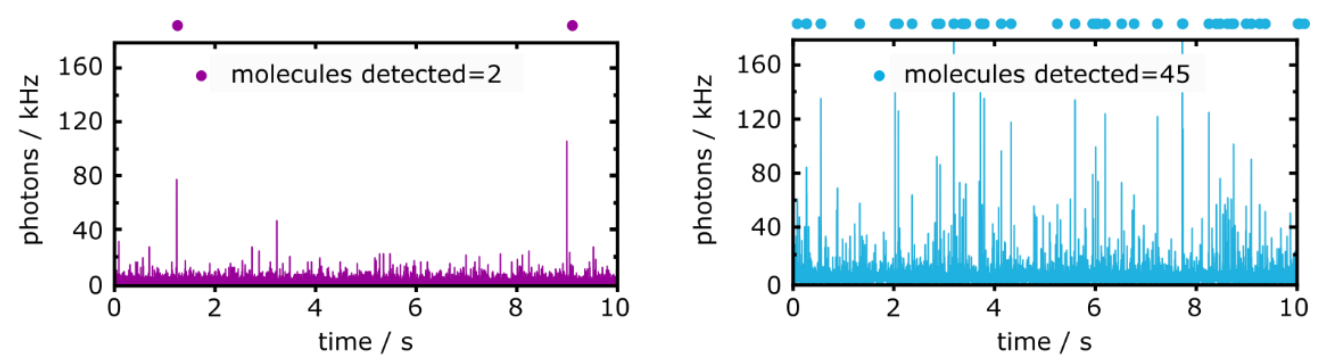

Figure 5: DigitISA platform used for the detection of weak biomolecular binding interactions on the example of $\alpha$-synuclein fibrils and their aptamer probe. (a) The binding of the aptamer probe to $\alpha$-synuclein fibrils reduces the electrophoretic mobility of the probe, allowing for discrimination between probe-bound and unbound species. (b) Electropherogram (left panel) as obtained by stepwise scanning of the confocal volume across the cross-section of the channel for the $\alpha$-synuclein fibrils-aptamer sample (blue line; average of $N=3$ repeats, the shaded bands correspond to the standard deviation) and for the free aptamer probe (purple line). The right panel shows a zoom-in region of the electropherogram. The shaded region in grey where the concentration of the complex exceeded that of the free probe 
$(1150 \mu \mathrm{m}<x<2000 \mu \mathrm{m})$ was used to estimate the concentration of the fibrils (see main text). Note, the photon arrival frequency in the region where $x<1100 \mu \mathrm{m}$ (i.e., where the probe elutes) was too high to count molecules one-by-one, hence the detected number of molecules in this region should be viewed as an approximation. (c) Exemplary photon-count time traces for the control sample (left panel, purple) and the mixture (right panel, blue) at the position indicated with colored dots in panel b (right). The number of molecules at each of the scanned positions was estimated using a burst-search algorithm as detailed in the Methods section. Dots above the time traces indicate detected single-molecule events. The bin time was $1 \mathrm{~ms}$ in all traces.

To demonstrate this capability, we incubated a sample of the fibrils with $10 \mathrm{nM}$ of aptamer probe (see Methods) and acquired an electropherogram across the cross-section of the device while applying a potential difference of $80 \mathrm{~V}$ across its terminals (Figure 5b, blue line). By comparison of the electropherogram for the aptamer-fibril sample relative to the aptamer-only control (Figure 5b, purple line), fibril-aptamer complexes could be identified at lower electrophoretic mobilities relative to the unbound aptamer peak. We estimated there to be a total of $\bar{n}_{\text {mixture }}=2066 \pm 47$ molecules for the aptamer-fibril mixture and $\bar{n}_{\text {control }}=926 \pm 132$ molecules of the unbound aptamer eluting in the shaded grey region highlighted in Figure $5 \mathrm{~b}$ $(1150 \mu \mathrm{m}<x<2100 \mu \mathrm{m})$ over a 10 -second timescale (see exemplary time traces in Figure $5 \mathrm{c}$ ). Using a similar analysis as before, these molecular counts yielded the concentration of proteinbound probe to be $18.7 \pm 2.3 \mathrm{pM}$ for the $\alpha$-synuclein fibrils, with a calculated LOD of $6.5 \mathrm{pM}$ The concentration of protein-bound probe corresponds to a total fibril binding site concentration of $1.0-1.9 \mathrm{nM}$ for a $K_{\mathrm{d}}$ range of $500-1000 \mathrm{nM}$.

We note that for a theoretical ELISA experiment with the same analyte concentration and a probe concentration of $1 \mathrm{nM}$, approximately only $1.9-3.8 \mathrm{pM}$ of fibril would be captured on the surface. Subsequent, rapid dissociation of the probe-aptamer complex due to the weak probe binding strength over the assay timescale would reduce the concentration of bound target well below the assay detection limit. Together, these factors illustrate how the DigitISA platform can be used for the detection of weak biomolecular binding interactions - a characteristic that is 
challenging to achieve with conventional multi-step approaches operating over longer time scales.

Additionally, our findings demonstrate the high sensitivity afforded by single-molecule detection of the protein-bound probe under flow, with LODs calculated to be in the low picomolar to sub-picomolar range for all of the examples shown. However, sensitivity could be improved yet further by optimizing the region of data acquisition to maximize the signal-tobackground ratio (i.e., $\bar{n}_{\text {mixture }} / \bar{n}_{\text {control }}$ ) and minimize $s_{\text {control }}$. For example, altering the acquisition window for the fibril-sensing experiment to between $1600 \mu \mathrm{m}<x<2100 \mu \mathrm{m}$ affords a $~ 10$-fold reduction in LOD from $6.5 \mathrm{pM}$ to $0.55 \mathrm{pM}$, although such an approach would require comparison of the observed $\bar{n}_{\text {control }}$ with calibration measurements to allow absolute quantification.

\section{Conclusion}

By combining microchip electrophoretic separation with single-molecule detection, we have demonstrated here a surface- and calibration-free platform for the digital detection and quantification of protein targets in solution. In contrast to incumbent assays that rely on surfaceimmobilization and serial washing and incubation steps, the DigitISA platform operates entirely in solution, does not require washing steps, and performs protein detection with single-molecule sensitivity in a single step using only a single affinity reagent. The assay format further combines affinity selection with physical separation and thus provides an additional criterion for target detection to afford high specificity and selectivity in the sensing process. Hence, DigitISA represents a fundamentally new route to surface-free specificity, increased sensitivity, and reduced complexity in state-of-the-art protein detection and biomedical analysis.

Crucially, because DigitISA provides the opportunity of in-solution analyte capture and fast removal of excess probe, arbitrarily high concentrations of binding probes can be employed. This enables quantitative analyte-capture regardless of capture probe affinity and further allows 
the probe-analyte binding interaction to be maintained during the entire sensing process. Together, these factors allow the use of relatively weak-binding capture reagents $\left(K_{\mathrm{d}}=10\right.$ $1000 \mathrm{nM}$ ), in contrast to conventional approaches, which require highly-optimized probes with sub-nanomolar affinity such as ultra-high-affinity antibody, whose production is non-trivial and not feasible for some biomarker targets. This finding is significant as it opens up the possibility to use commonly overlooked affinity reagents, such as aptamers, in quantitative single-molecule sensing, the production of which through SELEX ${ }^{30-32}$ and chemical synthesis is fast and inexpensive.

In the applications demonstrated here, by integrating the number of analytes that elute on chip as a function of time, our platform achieves calibration-free quantitation with subpicomolar LODs comparable to that of 'gold-standard' ELISAs. ${ }^{8}$ We predict that the sensitivity of DigitISA can be improved yet further by increasing the integration time in data acquisition. According to Poisson sampling statistics, longer acquisition times decrease the magnitude of the standard deviation of the sample relative to the mean and thus reduce detection limits of the assay, providing a promising route towards facile protein sensing with femtomolar sensitivity. Future iterations of the platform could also incorporate, for example, multicolor single-molecule spectroscopy and FRET techniques, ${ }^{39-41}$ as well as other microfluidic separation modalities ${ }^{42,43}$ combined with downstream analyses, ${ }^{44}$ to enhance assay parallelization, sensitivity, and robustness to experimental noise.

In summary, the microchip DigitISA platform presented herein constitutes a new experimental paradigm for protein biomarker sensing. The implementation of a fundamentally novel design principle releases constraints of conventional immunosensing approaches in terms of the thermodynamic and kinetics of the immunoprobe-analyte interaction and, uniquely, allows protein targets to be detected and their abundance to be directly quantified in solution within seconds and without the need of washing steps. We anticipate that the DigitISA 
methodology will find broad applicability to the detection and quantification of a wide range of biomolecular and biomedically relevant targets, thus paving the way for DigitISA to become a standard tool for highly sensitive biomolecular analysis and diagnostics.

\section{Methods}

DigitISA setup. The DigitISA optofluidic platform integrates microchip free-flow electrophoresis with single-molecule confocal fluorescence microscopy. Schematics of the microfluidic separation device, the optical setup, and their integration are shown in Figure 2. Briefly, the electrophoresis device is fabricated in PDMS by standard soft-lithography and molding techniques and bonded to a glass coverslip (see below). Fluids are injected into the chip via polyethylene tubing from glass syringes (Hamilton) and solution flow rates are controlled by automated syringe pumps (Cetoni neMESYS). Fluid waste is guided out of the device by tubing inserted into device outlets. Importantly, the free-flow electrophoresis functionality of the device is attained by a design that makes use of liquid electrolyte electrodes, ${ }^{21,22}$ where an electric potential is applied outside and downstream of the microfluidic separation chamber in separate electrolyte channels, and thereby allowed to propagate back to the separation area through the use of a co-flowing, highly conductive electrolyte solution (i.e., $3 \mathrm{M} \mathrm{KCl}$ ). This capacity is realized in the chip architecture by connecting the main electrophoresis chamber, which harbors the sample and the flanking buffered carrier medium from both sides, with the co-following electrolyte channels via narrow bridges. These connectors control the transfer of the electrolyte to the electrophoresis chamber and allow a thin sheet of electrolyte to form at and flow along both edges of the chamber, acting as liquid electrodes. Electric potentials are applied from a power supply (EA-PS 9500-06, EA ElektroAutomatik) via hollow metal dispensing tips (20G, Intertonics) inserted into the electrolyte outlets and carried into the device via the electrolyte solution, resulting in an electric field that spans across the main separation channel. Crucially, the use of liquid electrodes allows any 
generated electrolysis products to be flushed out of the chip and Joule heating to be reduced, permitting strong electric fields to be applied in a stable manner. This feature is of utmost importance for effective and high-performance electrophoretic separation. ${ }^{21-23}$

The optical unit of the DigitISA platform is based on a laser-induced fluorescence confocal microscope optimized for microfluidic experiments. The electrophoresis chip is secured to a motorized scanning stage (PZ-2000FT, Applied Scientific Instrumentation (ASI)), which is mounted onto a 'rapid automated modular microscope' (RAMM) frame (ASI). The motorized $x, y, z$-stage is equipped with a $z$-piezo for controlling precise sample placement along the optical axis of the microscope. To excite the sample in the device, the beam of a $488-\mathrm{nm}$ wavelength laser (Cobolt 06-MLD, $200 \mathrm{~mW}$ diode laser, Cobolt) is passed through a single-mode optical fiber (P3-488PM-FC-1, Thorlabs) and collimated at the exit of the fiber by an achromatic collimator (60FC-L-4-M100S-26, Schäfter + Kirchhoff) to form a beam with a Gaussian profile. The beam is then directed into the microscope, reflected by a dichroic beamsplitter (Di03-R488/561, Semrock), and subsequently focused to a concentric diffraction-limited spot in the microfluidic channel through a 60x-magnification water-immersion objective (CFI Plan Apochromat WI 60x, NA 1.2, Nikon). The emitted light from the sample is collected via the same objective, passed through the dichroic beamsplitter, and focused by achromatic lenses through a $30-\mu \mathrm{m}$ pinhole (Thorlabs) to remove any out-of-focus light. The emitted photons are filtered through a band-pass filter (FF01-520/35-25, Semrock) and then focused onto a singlephoton counting avalanche photodiode (APD, SPCM-14, PerkinElmer Optoelectronics), which is connected to a TimeHarp260 time-correlated single photon counting unit (PicoQuant).

Fabrication of the electrophoretic device. The microfluidic device was designed using AutoCAD software (Autodesk) and printed on acetate transparencies (Micro Lithography Services). The replica mold for fabricating the device was prepared through a single, standard soft-lithography step ${ }^{45,46}$ by spinning SU-8 3025 photoresist (MicroChem) onto a polished 
silicon wafer to a height of around $25 \mu \mathrm{m}$. The UV exposure step was performed with a custombuilt LED-based apparatus ${ }^{47}$ and the precise height of the features were measured to be $28 \mu \mathrm{m}$ by a profilometer (Dektak, Bruker). The mold was then used to generate a patterned PDMS slab. To this effect, the mold was casted in a 10:1 (w/w) mixture of PDMS (Dow Corning) and curing agent (Sylgard 184, Dow Corning), degassed and baked for $1.5 \mathrm{~h}$ at $65^{\circ} \mathrm{C}$. The formed PDMS slab was cut and peeled off the master and access holes for the inlet tubes were introduced using biopsy punches. The devices were then bonded to a thin glass coverslip after both the PDMS and the glass surface had been activated through oxygen plasma (Diener electronic, $40 \%$ power for $15 \mathrm{~s}$ ). Before injecting the solutions into the channels, the chips were exposed to an additional plasma oxidation step (80\% power for $500 \mathrm{~s}$ ) which rendered the channel surfaces more hydrophilic. ${ }^{48}$

Preparation of protein samples. The complex between the biotinylated and fluorophoreconjugated DNA sequence (5'-Atto488-CGACATCTAACCTAGCTCACTGAC-Biotin-3', HLPC purified; Biomers) and monovalent streptavidin was formed by mixing $25 \mathrm{pM}$ of the monovalent streptavidin sample with $50 \mathrm{pM}$ of biotinylated probe DNA in $10 \mathrm{mM}$ HEPES (pH 7.4) buffer (Sigma) supplemented with $0.05 \%$ Tween-20 (Thermo Scientific). Prior to its injection to the chip, the mixture was incubated at room temperature for 5 minutes. The monovalent streptavidin sample was kindly provided by the Howarth Lab (University of Oxford).

Recombinant IgE Kappa (clone AbD18705_IgE) was purchased from Bio-Rad Laboratories and dissolved to a concentration of $40 \mathrm{nM}$ in $10 \mathrm{mM}$ HEPES ( $\mathrm{pH}$ 7.4) buffer supplemented with $0.05 \%$ Tween-20. The protein sample was then mixed with the aptamer probe (5'-Atto488TGGGGCACGTTTATCCGTCCCTCCTAGTGGCGTGCCCC-3', HPLC purified; Integrated DNA Technologies (IDT)) diluted to a concentration of $50 \mathrm{pM}$ and incubated for 10 minutes before the experiment at room temperature prior to their injection to the chip. 
$\alpha$-synuclein fibrils were detected using T-SO508 aptamer ${ }^{37}$ (5'-Alexa488TTTTGCCTGTGGTGTTGGGGCGGGTGCG-3', HPLC purified; IDT). Prior to its use, the aptamer $\left(100 \mu \mathrm{M}\right.$ stock in $1 \mathrm{X}$ TE buffer) was heated to $70^{\circ} \mathrm{C}$ and cooled to room temperature to facilitate correct folding. The $\alpha$-synuclein fibrils were prepared by incubating $\alpha$-synuclein monomer as described earlier ${ }^{49,50}$ and sonicated (10\% power, $30 \%$ cycles for $1 \mathrm{~min}$; Sonopuls HD 2070, Bandelin). Following sonication, the fibrils were spun down and re-suspended in $10 \mathrm{mM}$ HEPES ( $\mathrm{pH} 7.4), 0.05 \%$ Tween-20. The aptamer and the fibrils were then mixed by suspending them into $10 \mathrm{mM}$ HEPES (pH 7.4) buffer supplemented with $0.05 \%$ Tween-20 to final concentrations of $10 \mathrm{nM}$ and $80 \mathrm{nM}$ (monomer equivalent), respectively. The mixture was incubated for 10 minutes before its injection to the chip.

Experimental procedures. For experiments performed on the biotin-streptavidin system, the sample and the co-flowing buffer were injected into the microfluidic device at a flow rate of 70 and $2000 \mu \mathrm{L} \mathrm{h}^{-1}$, respectively, and the $3 \mathrm{M} \mathrm{KCl}$ electrolyte solution from each of its inlets at $300 \mu \mathrm{L} \mathrm{h}^{-1}$ using glass syringes. For DigitISA experiments on $\operatorname{IgE}$ and on the $\alpha$-synuclein fibrils, these injection flow rates were 100, 1200, $400 \mu \mathrm{L} \mathrm{h}^{-1}$ and 50,1200, $200 \mu \mathrm{L} \mathrm{h}^{-1}$, respectively. The PDMS-glass chip was secured to the motorized, programmable microscope stage and once a stable flow in the device had been established, a potential difference across the device was applied. The photon-count time traces were obtained by translocating the microscope stage across the cross-section (Figure 2, step-scan line is indicated) using a customwritten Python script that simultaneously controlled the stage movement and the data acquisition at a distance of $4 \mathrm{~mm}$ downstream from where the electric field was first applied and at mid-height of the device (i.e., $\sim 14 \mu \mathrm{m}$ above the surface of the glass coverslip). Each of the experiments was performed in a freshly fabricated PDMS device and simultaneous current readings were taken to ensure that the efficiencies did not vary between the devices and comparisons could be drawn between the deflected distances at which molecules eluted. The 
laser power at the back aperture of the objective was adjusted to $150 \mu \mathrm{W}$ in all experiments. Photon recordings were done in T2 mode and the arrival times of photons were measured in respect to the overall measurement start with 16-ps resolution.

Data analysis. The passing molecules were identified and distinguished from the background by requiring the IPT to remain short for the arrival of a number of consecutive photons. This approach has been shown to allow effective discrimination between background photons and those that originate from a fluorescently labeled molecules passing the laser spot and emitting photons..$^{25,26,51,52}$ Specifically, an IPT threshold of $100 \mu \mathrm{s}$ was used for the analysis of the biotinstreptavidin interaction and the IgE sample with consecutive photon arrival events identified as a molecule when a packet of at least 7 photons arrived each with an IPT below threshold. For the analysis of the fibril sample these thresholds were set to $5 \mu$ s and 30 photons, respectively. In all cases, before analysis the IPT traces were processed with Lee filter $(n=4)^{53}$ to smoothen regions of constant signal while keeping those with rapid parameter changes, such as the edges of the bursts unaffected.

Dimensions of the confocal volume from FCS. The dimensions of the confocal volume were determined by performing an FCS experiment on Atto488 carboxylic acid (100 pM; $150 \mu \mathrm{W}$ laser power). Using its diffusion coefficient of $D=400 \mu \mathrm{m}^{2} \mathrm{~s}^{-1}$, the effective volume of the confocal volume was evaluated to be $V_{\text {eff }}=4.2 \mathrm{fL}$ and the kappa factor to be $\kappa=6.0$, yielding an estimate for the dimensions of the confocal volume as $z=3 \mu \mathrm{m}$ in its height and around $w=0.4 \mu \mathrm{m}$ in its width. The correlation analysis was done using the SymPhoTime 64 software package (Picoquant).

Simulation of probe-target binding interaction. Estimations of analyte binding and complex dissociation depicted in Figures $1 \mathrm{~b}$ were obtained by examining the binding of probe $(\mathrm{P})$ to its target analyte (A) according to: 


$$
\mathrm{P}+\mathrm{A} \underset{k_{\mathrm{off}}}{\stackrel{k_{\mathrm{on}}}{\rightleftharpoons}} \mathrm{PA}
$$

where $k_{\text {on }}$ and $k_{\text {off }}$ are the rate constants for the formation of the complex and its dissociation, respectively. The simulations in Figure $1 \mathrm{~b}(\mathrm{i})$ describe the thermodynamic equilibrium for probes with various dissociation constants $K_{\mathrm{d}}$ ranging from $0.1 \mathrm{nM}$ to $1000 \mathrm{nM}$ as a function of its concentration $c_{\text {probe }}$. The simulations in Figure $1 \mathrm{~b}(\mathrm{ii})$ illustrate the dissociation of the proteinanalyte complex after the removal of the excess reagents relative to the amount of complex that was present at equilibrium. Any changes in the dissociation constant $\left(K_{\mathrm{d}}=k_{\text {off }} / k_{\text {on }}\right)$ were assumed to originate from alterations in the rate constant that govern the dissociation of the complex $\left(\mathrm{PA} \stackrel{k_{\text {off }}}{\rightarrow} \mathrm{P}+\mathrm{A}\right)$ rather than changes in the rate constant governing the formation of the complex $\left(\mathrm{P}+\mathrm{A} \stackrel{k_{\mathrm{on}}}{\rightarrow} \mathrm{PA}\right)$. The rate constant for the latter reaction varies between probes. ${ }^{54}$ Here we used $k_{\text {on }}=10^{-4} \mathrm{M}^{-1} \mathrm{~s}^{-1}$, which for a fixed $K_{\mathrm{d}}$ yields conservative estimates for $k_{\mathrm{off}}$, and hence, for the rate at which the complex dissociates.

\section{Conflict of interest}

Parts of this work have been the subject of a patent application filed by Cambridge Enterprise Limited, a fully owned subsidiary of the University of Cambridge.

\section{Acknowledgements}

The authors thank the Howarth Lab (University of Oxford) for monovalent streptavidin samples. The research leading to these results has received funding from the European Research Council under the European Union's Horizon 2020 Framework Programme through the Marie Skłodowska-Curie grant MicroSPARK (agreement no. 841466; G.K.), the Herchel Smith Fund of the University of Cambridge (G.K.), the Wolfson College Junior Research Fellowship (G.K.), the Schmidt Science Fellows program in partnership with the Rhodes Trust (K.L.S.), the Engineering and Physical Sciences Research Council (K.L.S), St. John's College. (K.L.S), the 
EPSRC Cambridge NanoDTC (EP/L015978/1; W.E.A.), the European Union Horizon 2020 research and innovation programme (ETN grant 674979-NANOTRANS; Q.P.), the European Research Council under the European Union's Seventh Framework Programme (FP7/20072013) through the ERC grants PhysProt (agreement no. 337969; T.P.J.K), and the Newman Foundation (T.P.J.K.).

\section{Author contributions}

G.K., K.L.S., W.E.A., and T.P.J.K. conceptualized the study and devised the methodology, G.K., K.L.S., P.C., C.G.T., and D.K. contributed experimental hardware and expertise, G.K. and W.E.A. performed the investigation, G.K., K.L.S., W.E.A., R.P.B.J., and Q.P. performed the analysis, and R.P.B.J. and Q.P. contributed relevant software. G.K., K.L.S., and W.E.A. wrote the original draft and all other authors reviewed and edited it.

\section{References}

1. Galasko, D. Biomarkers for Alzheimer's disease - Clinical needs and application. $J$. Alzheimer's Dis. 8, 339-346 (2005).

2. Meriggioli, M. N. Use of immunoassays in neurological diagnosis and research. Neurol. Res. 27, 734-740 (2005).

3. Srinivas, P. R., Kramer, B. S. \& Srivastava, S. Trends in biomarker research for cancer detection. Lancet Oncol. 2, 698-704 (2001).

4. Rifai, N., Gillette, M. A. \& Carr, S. A. Protein biomarker discovery and validation: the long and uncertain path to clinical utility. Nat. Biotechnol. 24, 971-83 (2006).

5. Kelley, S. O. et al. Advancing the speed, sensitivity and accuracy of biomolecular detection using multi-length-scale engineering. Nat. Nanotechnol. 9, 969-80 (2014).

6. Wild, D. The immunoassay handbook. Theory and Applications of Ligand Binding, 
ELISA and Related Techniques. (Elsevier Ltd., 2013).

7. Wilson, R. Sensitivity and specificity: twin goals of proteomics assays. Can they be combined? Expert Rev. Proteomics 10, 135-149 (2013).

8. Giljohann, D. A. \& Mirkin, C. A. Drivers of biodiagnostic development. Nature 462, $461-464$ (2009).

9. Engvall, E. \& Perlmann, P. Enzyme-linked immunosorbent assay (ELISA) quantitative assay of immunoglobulin G. Immunochemistry 8, 871-874 (1971).

10. Lequin, R. M. Enzyme immunoassay (EIA)/enzyme-linked immunosorbent assay (ELISA). Clin. Chem. 51, 2415-2418 (2005).

11. Sano, T., Smith, C. L. \& Cantor, C. R. Immuno-PCR: very sensitive antigen detection by means of specific antibody-DNA conjugates. Science 258, 120-2 (1992).

12. De La Rica, R. \& Stevens, M. M. Plasmonic ELISA for the ultrasensitive detection of disease biomarkers with the naked eye. Nat. Nanotechnol. 7, 821-824 (2012).

13. Stern, E. et al. A nanoelectronic enzyme-linked immunosorbent assay for detection of proteins in physiological solutions. Small 6, 232-8 (2010).

14. Shim, J. et al. Ultrarapid Generation of Femtoliter Microfluidic Droplets for SingleMolecule-Counting Immunoassays. ACS Nano 7, 5955-5964 (2013).

15. Rissin, D. M. et al. Single-molecule enzyme-linked immunosorbent assay detects serum proteins at subfemtomolar concentrations. Nat. Biotechnol. 28, 595-599 (2010).

16. Geiss, G. K. et al. Direct multiplexed measurement of gene expression with colorcoded probe pairs. Nat. Biotechnol. 26, 317-325 (2008).

17. Gold, L. et al. Aptamer-Based Multiplexed Proteomic Technology for Biomarker Discovery. PLoS One 5, e15004 (2010). 
18. Chang, L. et al. Single molecule enzyme-linked immunosorbent assays: Theoretical considerations. J. Immunol. Methods 378, 102-115 (2012).

19. Sormanni, P., Aprile, F. A. \& Vendruscolo, M. Third generation antibody discovery methods: in silico rational design. Chem. Soc. Rev. 47, 9137-9157 (2018).

20. Knowles, T. P. J., Vendruscolo, M. \& Dobson, C. M. The amyloid state and its association with protein misfolding diseases. Nat. Rev. Mol. Cell Biol. 15, 384-396 (2014).

21. Arter, W. E. et al. Combining Affinity Selection and Specific Ion Mobility for Microchip Protein Sensing. Anal. Chem. 90, 10302-10310 (2018).

22. Saar, K. L. et al. On-chip label-free protein analysis with downstream electrodes for direct removal of electrolysis products. Lab Chip 18, 162-170 (2018).

23. Saar, K. L., Müller, T., Charmet, J., Challa, P. K. \& Knowles, T. P. J. Enhancing the Resolution of Micro Free Flow Electrophoresis through Spatially Controlled Sample Injection. Anal. Chem. 90, 8998-9005 (2018).

24. Howarth, M. et al. A monovalent streptavidin with a single femtomolar biotin binding site. Nat. Methods 3, 267-273 (2006).

25. Fries, J. R., Brand, L., Eggeling, C., Köllner, M. \& Seidel, C. A. M. Quantitative identification of different single molecules by selective time-resolved confocal fluorescence spectroscopy. J. Phys. Chem. A 102, 6601-6613 (1998).

26. Schaffer, J. et al. Identification of Single Molecules in Aqueous Solution by TimeResolved Fluorescence Anisotropy. J. Phys. Chem. A 103, 331-336 (1999).

27. Grimbacher, B., Holland, S. M. \& Puck, J. M. Hyper-IgE syndromes. Immunol. Rev. 203, 244-50 (2005). 
28. Ogawa, M., Kochwa, S., Smith, C., Ishizaka, K. \& McIntyre, O. R. Clinical aspects of IgE myeloma. N. Engl. J. Med. 281, 1217-1220 (1969).

29. Wiegand, T. W. et al. High-affinity oligonucleotide ligands to human IgE inhibit binding to Fc epsilon receptor I. J. Immunol. 157, 221-30 (1996).

30. Tuerk, C. \& Gold, L. Systematic evolution of ligands by exponential enrichment: RNA ligands to bacteriophage T4 DNA polymerase. Science 249, 505-10 (1990).

31. Ellington, A. D. \& Szostak, J. W. In vitro selection of RNA molecules that bind specific ligands. Nature 346, 818-822 (1990).

32. Zhuo, Z. et al. Recent Advances in SELEX Technology and Aptamer Applications in Biomedicine. Int. J. Mol. Sci. 18, 2142 (2017).

33. Lollo, B., Steele, F. \& Gold, L. Beyond antibodies: New affinity reagents to unlock the proteome. Proteomics 14, 638-644 (2014).

34. Turgeon, R. T., Fonslow, B. R., Jing, M. \& Bowser, M. T. Measuring aptamer equilibria using gradient micro free flow electrophoresis. Anal. Chem. 82, 3636-3641 (2010).

35. German, I., Buchanan, D. D. \& Kennedy, R. T. Aptamers as ligands in affinity probe capillary electrophoresis. Anal. Chem. 70, 4540-4545 (1998).

36. Campbell, J., Pollock, N. R., Sharon, A. \& Sauer-Budge, A. F. Development of an automated on-chip bead-based ELISA platform. Anal. Methods 7, 8472-8477 (2015).

37. Tsukakoshi, K., Abe, K., Sode, K. \& Ikebukuro, K. Selection of DNA aptamers that recognize $\alpha$-synuclein oligomers using a competitive screening method. Anal. Chem. 84, 5542-5547 (2012).

38. Parnetti, L. et al. CSF and blood biomarkers for Parkinson's disease. The Lancet 
Neurology 18, 573-586 (2019).

39. Streets, A. M. \& Huang, Y. Microfluidics for biological measurements with singlemolecule resolution. Curr. Opin. Biotechnol. 25, 69-77 (2014).

40. Kim, S. et al. High-throughput single-molecule optofluidic analysis. Nat. Methods 8 , $242-5(2011)$.

41. Wunderlich, B. et al. Microfluidic mixer designed for performing single-molecule kinetics with confocal detection on timescales from milliseconds to minutes. Nat. Protoc. 8, 1459-1474 (2013).

42. Arter, W. E., Levin, A., Krainer, G. \& Knowles, T. P. J. Microfluidic approaches for the analysis of protein-protein interactions in solution. Biophys. Rev. 1-11 (2020). doi:10.1007/s12551-020-00679-4

43. Herling, T. W., Levin, A., Saar, K. L., Dobson, C. M. \& Knowles, T. P. J. Microfluidic approaches for probing amyloid assembly and behaviour. Lab Chip 18, 999-1016 (2018).

44. Saar, K. L. et al. Rapid two-dimensional characterisation of proteins in solution. Microsystems Nanoeng. 5, 1-10 (2019).

45. Duffy, D. C., McDonald, J. C., Schueller, O. J. A. \& Whitesides, G. M. Rapid prototyping of microfluidic systems in poly(dimethylsiloxane). Anal. Chem. 70, 4974 4984 (1998).

46. Arter, W. E. et al. Digital Sensing and Molecular Computation by an Enzyme-Free DNA Circuit. ACS Nano (2020). doi:10.1021/acsnano.0c00628

47. Challa, P. K., Kartanas, T., Charmet, J. \& Knowles, T. P. J. Microfluidic devices fabricated using fast wafer-scale LED-lithography patterning. Biomicrofluidics 11, 
014113 (2017).

48. Tan, S. H., Nguyen, N.-T., Chua, Y. C. \& Kang, T. G. Oxygen plasma treatment for reducing hydrophobicity of a sealed polydimethylsiloxane microchannel. Biomicrofluidics 4, 032204 (2010).

49. Chen, S. W. et al. Structural characterization of toxic oligomers that are kinetically trapped during $\alpha$-synuclein fibril formation. Proc. Natl. Acad. Sci. U. S. A. 112, E1994E2003 (2015).

50. Arter, W. E. et al. Rapid Fractionation and Characterisation of Alpha-Synuclein Oligomers in Solution. bioRxiv 2020.03.10.985804 (2020). doi:10.1101/2020.03.10.985804

51. Krainer, G., Hartmann, A. \& Schlierf, M. FarFRET: Extending the Range in SingleMolecule FRET Experiments beyond $10 \mathrm{~nm}$. Nano Lett. 15, (2015).

52. Krainer, G. et al. A minimal helical-hairpin motif provides molecular-level insights into misfolding and pharmacological rescue of CFTR. Commun. Biol. 1, 154 (2018).

53. Enderlein, J., Robbins, D. L., Ambrose, W. P. \& Keller, R. A. Molecular Shot Noise, Burst Size Distribution, and Single-Molecule Detection in Fluid Flow: Effects of Multiple Occupancy. J. Phys. Chem. A 102, 6089-6094 (1998).

54. Landry, J. P., Ke, Y., Yu, G. L. \& Zhu, X. D. Measuring affinity constants of 1450 monoclonal antibodies to peptide targets with a microarray-based label-free assay platform. J. Immunol. Methods 417, 86-96 (2015). 